\title{
A Model of the Wind Direction Signature in the Stokes Emission Vector from the Ocean Surface at Microwave Frequencies
}

\author{
Steven C. Reising ${ }^{(1)}$ and Adriano J. Camps ${ }^{(2)}$ \\ (1) Microwave Remote Sensing Laboratory, University of Massachusetts, Amherst, MA 01003 \\ Ph: (413) 577-0697; Fax: (413) 545-4652; Email: reising@ecs.umass.edu \\ (2) Department of Signal Theory and Communications, Universitat Politècnica de Catalunya, \\ Campus Nord, D4, 08034 Barcelona, SPAIN \\ Ph: 34-93.401.60.85; Fax: 34-93.401.72.32; Email: camps@tsc.upc.es
}

\begin{abstract}
This paper presents a model of the Stokes vector emission from the ocean surface. The ocean surface is described as an ensemble of facets with Cox and Munk's Gram-Charlier slope distribution. The study discusses the impact of different up-wind and cross-wind rms slopes, skewness, peakedness, foam cover models and atmospheric effects on the azimuthal variation of the Stokes vector, as well as the limitations of the model. Simulation results compare favorably, both in mean value and azimuthal dependence, with SSM/I data at $53^{\circ}$ incidence angle and with JPL's WINDRAD measurements at incidence angles from $30^{\circ}$ to $65^{\circ}$, and at wind speeds from 2.5 to $11 \mathrm{~m} / \mathrm{s}^{\text {. }}$
\end{abstract}

\section{INTRODUCTION}

Wentz [1] found small wind direction signatures in SSM/I vertical and horizontal brightness temperatures. Recent experimental evidence seems to indicate that the wind direction dependence of the third Stokes parameter $(U)$ is more robust with respect to atmospheric effects, preserving its shape and zero crossings in azimuth.

Several approaches for computing the azimuthal emission signature of a rough surface are found in the literature: the two-scale/small perturbation model (SPM) using Bragg scattering [2], the small-slope expansion, Monte Carlo simulations using geometric optics (GO) [3], and the method of moments. Strictly speaking, SPM is accurate at low frequencies ( $k \sigma \leq 0.3, k$ : wavenumber, $\sigma$. rms height), while $\mathrm{GO}$ is accurate at high frequencies. Two-scale models have been successfully applied to model the polarimetric emission of the ocean surface, but they rely on the choice of a proper cut-off wavenumber $k_{d}$ to separate large-scale and small-scale waves. As pointed out by Noll [4], for frequencies above 20 $\mathrm{GHz}$ "the selection of the cut-off wavenumber $k_{d}$ becomes critical." To overcome this problem and the problems associated with numerical Monte Carlo simulations, the present model improves the analytical model [5] to include foam, skewness and peakedness effects, in addition to the

"Work performed during A. Camps' sabbatical at the Microwave Remote Sensing Laboratory, University of Massachusetts, Amherst. Fellowship from the Spanish Ministry of Education and Culture (PR98 0046131105). asymmetry between up-wind and cross-wind rms slope spectra. Atmospheric effects prove to be an important contribution, principally the polarization induced by the ocean surface when the downwelling radiation is scattered, especially at low wind speeds and at some observation angles.

\section{DESCRIPTION AND LIMITATIONS OF THE MODEL}

The polarimetric emission behavior of the sea surface is given by $[6]$ :

$$
\begin{aligned}
& e_{h}(\theta, \phi)=1-\frac{1}{4 \pi \cos \theta} \iint_{2 \pi} \cos \theta_{i} \quad \underset{v v v v}{\left(\gamma_{h h h h}+\gamma_{\nu h h v}\right)} d \Omega_{i}, \\
& e_{V}(\theta, \phi)=-\frac{1}{4 \pi \cos \theta} \iint_{2 \pi} \cos \theta_{i}^{\mathfrak{R} e}\left(\gamma_{v h h h}+\gamma_{v v h v}\right) d \Omega_{i} .
\end{aligned}
$$

The polarimetric bistatic scattering coefficients $\left(\gamma_{p q r s}\right)$ are derived following a procedure similar to Stogryn [5] with Wilheit's [7] frequency corrections for the rms slopes $\left(0.3+0.02 f_{[\mathrm{GHz}]}, f \leq 35 \mathrm{GHz}\right)$. However, the complete GramCharlier pdf [8] function is included to describe the ocean surface slopes. It is found that, at constant observation angle $\theta$, when including only the up-wind / cross-wind asymmetry $\left(\sigma_{u}^{2} \neq \sigma_{\mathrm{c}}^{2}\right), \mathrm{T}_{v}(\theta, \phi), \mathrm{T}_{h}(\theta, \phi)$ and $\mathrm{U}(\theta, \phi)$ exhibit only a second harmonic dependence on $\phi$. When skewness effects $\left(c_{21}, c_{03}\right)$ are included, the first harmonic in $\phi$ appears. The impact of the peakedness coefficients $\left(c_{40}, c_{22}\right.$ and $\left.c_{04}\right)$ is found to be much less important. The emissivity is computed analytically by averaging the Fresnel relationships over the distribution of wave slopes, including polarization effects and the projection of each facet. Multiple scattering and surface shadowing effects are not included in the model. The validity of the GO method applied to the ocean surface was analyzed by Kunkee and Gasiewski [3], who compared different criteria found in the literature. According to their results, at $37 \mathrm{GHz} \mathrm{GO}$ can be applied for all wind speeds up to $24 \mathrm{~m} / \mathrm{s}$ at $30^{\circ}$ observation angle, and up to about $9 \mathrm{~m} / \mathrm{s}$ at $60^{\circ}$ observation angle.

When only $\sigma_{u}^{2}$ and $\sigma_{\mathrm{c}}^{2}$ are considered, $\Delta \mathrm{T}_{\nu}(\theta, \phi)$ and $\Delta \mathrm{T}_{h}(\theta, \phi)$ have an azimuthal dependence as $\cos (2 \phi)$, while $\mathrm{U}(\theta, \phi)$ is proportional to $\sin (2 \phi)$. When pdf skewness is taken into account, a first harmonic dependence as $\cos (\phi)$ is observed for $\Delta \mathrm{T}_{\nu}(\theta, \phi)$ and $\Delta \mathrm{T}_{h}(\theta, \phi)$, and as $\sin (\phi)$ for $\mathrm{U}(\theta, \phi)$. Taking 
into account peakedness coefficients introduces a minor change in the shape of the azimuthal dependence. This can be seen in Fig. 1, showing the azimuthal dependence of $\Delta \mathrm{T}_{v}(\theta, \phi)$ at $53^{\circ}$ incidence angle and wind speed $7.9 \mathrm{~m} / \mathrm{s}$ when only up-wind/cross-wind asymmetry is considered (dotted line), when skewness effects are included (dashed line) and when peakedness effects are also included (solid line). Simulation results using $W u$ 's model [9] for $\sigma_{u}^{2}$ and $\sigma_{\mathrm{c}}^{2}$ are not significantly different from those using Cox and Munk's [8] relationships.

Two foam coverage models were analyzed in this study: a uniform cover, polarization insensitive model [7], and a dynamic foam model [3] with different vertical and horizontal foam emissivities [10]. Wilheit [7] suggested an isotropic reflectivity reduction by a factor $F \approx 0.006\left(1-\exp \left(-f_{\left[\mathrm{CH}_{2}\right]} / 7.5\right)\right)\left(w_{[m / s]}-7\right)$ for wind speeds larger than $w=7 \mathrm{~m} / \mathrm{s}$. Differences between both models are not found to be significant, as shown in Fig. 2 for $\Delta \mathrm{T}_{v}(\theta, \phi)$ at $53^{\circ}$ incidence angle and wind speed $12.2 \mathrm{~m} / \mathrm{s}$.

Other sources of uncertainty are atmospheric stability $\Delta T=T_{\text {water }}-T_{\text {atm }}$, water temperature $T_{\text {water }}$ (water viscosity changes with $T_{\text {water }}$, wind duration, fetch, and water salinity. Since there is no clear evidence indicating which model is better, in the following simulations Wilheit's simple model is used. Two main effects produced by the atmosphere have been reported in the literature: atmospheric stability and attenuation. The first one affects the wind speed height profile, and, for a given wind speed at a reference height, it changes the mean wind speed at the surface and in turn, the scattering coefficients [11]. On the other hand, atmospheric attenuation produces an effective depolarization of the radiation reaching the radiometer, and hence, a decrease in the amplitude of the harmonics of $\mathrm{T}_{h}, \mathrm{~T}_{v}$ and $\mathrm{U}$.

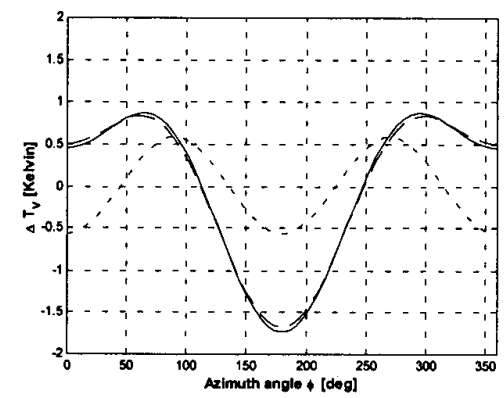

Figure 1. Simulated $\Delta \mathrm{T}_{\nu}(\theta, \phi)$ including up-wind/cross-wind asymmetry (dotted line), and skewness effects (dashed line) and peakedness effects (solid line). Other parameters: $53^{\circ}$ incidence angle, wind speed $=7.9 \mathrm{~m} / \mathrm{s}, \mathrm{SST}=12^{\circ} \mathrm{C}, \mathrm{SSS}=33 \mathrm{psu}$

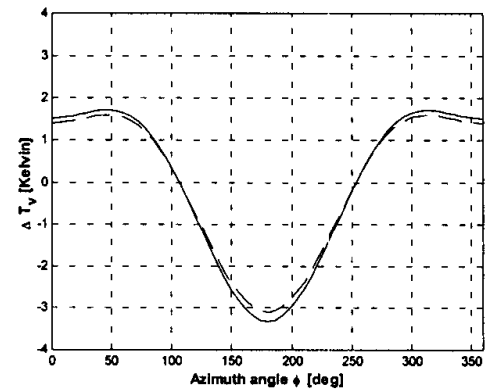

Figure 2. Sea foam effects: Wilheit's model (solid line) and Kunkee-Gasiewski dynamic model (dotted line)

A third effect that, to the authors' knowledge, has received little attention in the literature, is the induced polarization and azimuthal dependence that the total downwelling temperature (atmosphere + galactic + cosmic noise) acquires when it is scattered from the ocean surface and then is measured by the radiometer. Simulation results show that atmospheric effects can be significant at low wind speeds, i.e. when the surface reflectivity increases, and at certain observation angles at which the azimuthal signature of the emitted Stokes vector is minimum, around $45^{\circ}$ for horizontal polarization.

\section{COMPARISON WITH EXPERIMENTAL DATA}

Simulation results were compared with JPL's reported WINDRAD measurements obtained in circular flights at a constant observation angle [12]. To make the comparison more precise, because of the lack of information on the atmospheric conditions and the necessary corrections, we first compared azimuthally averaged values of WINDRAD measurements with the apparent brightness temperature computed using the present model, which includes: ocean surface emission, scattered downwelling atmospheric temperature, upwelling atmospheric temperature and atmospheric attenuation for a horizontally stratified clear atmosphere (US standard atmosphere). Since it appears that the data were not compensated for atmospheric effects nor for their azimuthal dependence, our numerical simulations include this contribution, as well as the atmospheric attenuation up to $10 \mathrm{Km}$ flight height, that obviously depends on frequency and observation angle.

Figure 3 shows the azimuthal dependence of $\Delta \mathrm{T}_{h}$ at $37 \mathrm{GHz}$, $45^{\circ}$ incidence angle and a wind speed of $9 \mathrm{~m} / \mathrm{s}$ when the downwelling atmospheric emission scattered over the ocean is included (solid line) or not (dotted line), showing a much better agreement with reported experimental data.

Figure 4 shows the azimuthal dependence of $\mathrm{T}_{v}, \mathrm{~T}_{h}$ and $\mathrm{U}$ with respect to the up-wind direction $\left(\phi=0^{\circ}\right)$, at observation angles $\theta=55^{\circ}$, for frequencies of $37 \mathrm{GHz}$ and $19 \mathrm{GHz}$, and at a wind speed of $9 \mathrm{~m} / \mathrm{s}$, for which foam effects have already appeared. The plots show that at $\theta=55^{\circ}$ and even at $65^{\circ}$, the azimuthal dependence is quite accurately predicted. It is dominated by the first harmonic in $\mathrm{T}_{v}$, and by the second harmonic in $T_{h}$. The agreement of $U$ is excellent at all observation angles and at both frequencies. 


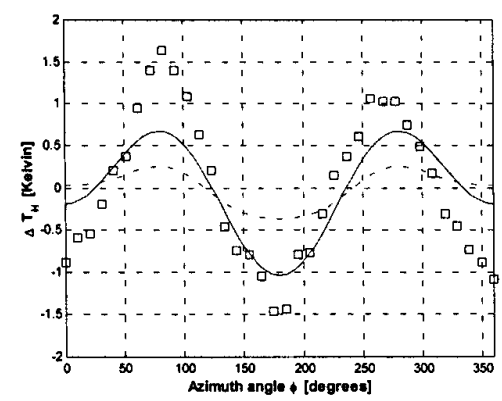

Figure 3. Atmospheric contribution to the azimuthal dependence of $\Delta \mathrm{T}_{h}$ at $37 \mathrm{GHz}$ at $\theta=45^{\circ}$ and $w=9 \mathrm{~m} / \mathrm{s}$. Dotted line: ocean emission attenuated by the atmosphere. Solid line: ocean emission and downwelling atmospheric temperature scattered over the ocean surface, attenuated by the atmosphere up to $10 \mathrm{~km}$ height.

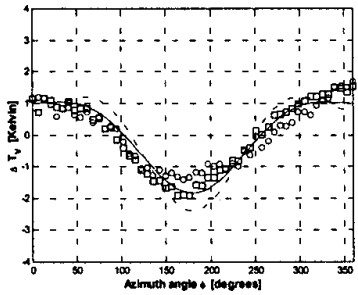

a)

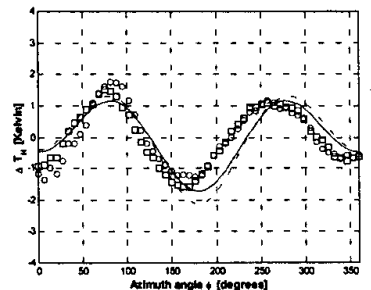

b)

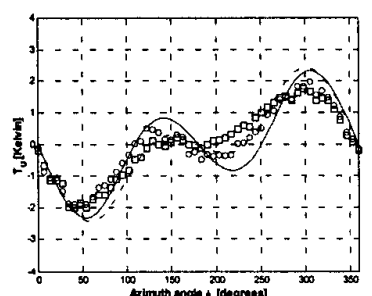

c)

Figure 6. Comparison of JPL's WINRAD measurements with numerical simulations. Data: O $19 \mathrm{GHz}, \square 37 \mathrm{GHz}$. Simulations: solid line $-19 \mathrm{GHz}$, dotted line $-37 \mathrm{GHz}$. Observation angles: $55^{\circ}$ $(a, b, c)$ and $65^{\circ}(d, e, f) . \mathrm{T}_{\nu}(a, d), \mathrm{T}_{h}(b, e)$, and $\mathrm{U}(c, f) . \mathrm{SST}=12^{\circ} \mathrm{C}$, SSS $=33 \mathrm{psu}$, wind speed $=9 \mathrm{~m} / \mathrm{s}$ and the US std. clear atmosphere.

\section{CONCLUSIONS}

This paper presents the results of an analytical model of the wind direction signature in the first three elements of the Stokes vector $\left(T_{h}, T_{v}\right.$ and $\left.U\right)$ of ocean surface emission. The model includes the complete Cox and Munk probability density function (pdf) of ocean surface slopes. The asymmetry of up-wind/cross-wind rms slopes is responsible for the second azimuth harmonic of the Stokes vector, while the first harmonic is dominated by skewness terms. Peakedness terms have a minor impact. The main advantages of this approach are its simplicity and its independence of the choice of any particular tuning parameter. This model is only valid up to a certain wind speed, for a given frequency and observation angle, e.g. for wind speeds up to $17 \mathrm{~m} / \mathrm{s}$ at 37 $\mathrm{GHz}$ at $53^{\circ}$ incidence angle. The importance of the polarization induced in the downwelling atmospheric brightness temperature by its scattering from the ocean surface proves to be non-negligible, especially for $T_{h}$ and $U$ at low wind speeds and/or incidence angles near $45^{\circ}$. The simulation results compare favorably with both JPL's WINDRAD measurements and Wentz's SSM/I geophysical function, both in the shape and amplitude of the azimuth variations of $\mathrm{T}_{h}, \mathrm{~T}_{v}$ and $\mathrm{U}$, and in their mean value.

\section{REFERENCES}

[1] Wentz, F.J., "Measurement of Oceanic Wind Vector Using Satellite Microwave Radiometers", IEEE Trans. on Geosci. and Remote Sens., Vol. 30, No. 5., pp. 960-972, September 1992.

[2] Yueh, S.H., "Modeling of Wind Direction Signals in Polarimetric Sea Surface Brightness Temperatures", IEEE Trans. on Geosci. and Remote Sens., Vol. 35, No. 6, pp. 1400-1418, November 1997.

[3] Kunkee, D.B. and A.J. Gasiewski, "Simulation of Passive Microwave Wind Direction Signatures over the Ocean using Asymmetric-Wave Geometrical Optics Model", Radio Sci., Vol. 32, No. 1, pp. 59-78, Jan.-Feb. 1997.

[4] Noll, J., "Small Perturbation Model Simulations Applied to POLRAD-96", Proceedings of the International Workshop on Polarimetric Radiation Experiment POLRAD '96, WPP-135, ESTEC, Noordwijk, The Netherlands, April 29, pp. 19-23, 1997.

[5] Stogryn, A., "The Apparent Temperature of the Sea at Microwave Frequencies", IEEE Trans. on Antennas and Propagation, AP-15, pp. 278-286, March 1967.

[6] Peake, W.H., "Interaction of Electromagnetic Waves with some Natural Surfaces", IRE Trans. on Antennas and Propagation (special supplement), Vol. 7, pp. S324-S329, 1959.

[7] Wilheit, T.T., "A Model for the Microwave Emissivity of the Ocean's Surface as a Function of Wind Speed", IEEE Trans. Geosci. Electr., Vol. 17, No. 4, pp. 244-249, 1979.

[8] Cox, C. and W. Munk, "Measurement of the Roughness of the Sea Surface from Photographs of the Sun's Glitter", Journal of the Optical Society of America, Vol. 44, No. 11, pp. 838-850, November 1954.

[9] Wu, J., "Mean Square Slopes of the Wind Disturbed Water Surface, Their Magnitude, Directionality, and Composition", Radio Sci., Vol. 25, No. 1, pp. 37-48, 1990.

[10] Smith, P.M., "The Emissivity of Sea Foam at 19 and 37 GHz", IEEE Trans. Geosci. and Remote Sens., Vol. 26, No. 5, pp. 541-547, 1988.

[11] Pospelov, M.N., "The Atmospheric Influence on SeaSurface Polarized Microwave Emission", Proceedings of the International Geosci. and Remote Sens. Symp., pp. 1135-1137, IEEE Press, Piscataway, NJ, 1996.

[12] Yueh, S.H., W.J. Wilson, F.K. Li, S.V. Nghiem, and W.B. Ricketts, "Polarimetric Brightness Temperatures of Sea Surfaces Measured with Aircraft K- and Ka-Band Radiometers", IEEE Trans. Geosci. and Remote Sens., Vol. 35, No. 5, pp. 1177-1187, September 1997. 